\title{
Mycobacterium avium subespecie paratuberculosis en heces de bovinos del municipio de Sincelejo, Sucre, Colombia
}

\author{
Mycobacterium avium subsp. paratuberculosis IN BOVINE FAECES FROM THE \\ Municipality of Sincelejo, Sucre, Colombia
}

Libardo Caraballo-Blanco ${ }^{1,3}$, Anais Castellar-Martínez ${ }^{1}$, Enrique Pardo-Pérez ${ }^{2}$

\section{Resumen}

\begin{abstract}
El objetivo del estudio fue determinar la presencia de Mycobacterium avium subspecie paratuberculosis (MAP) en heces de bovinos del municipio de Sincelejo, Sucre, Colombia. Se realizó un estudio bacteriológico directo por técnicas de microscopía y reacción en cadena de la polimerasa (PCR) para pooles de heces con dos marcadores moleculares, uno específico para MAP (IS900) y otro basado en 16S del género Mycobacterium. La presencia de bacilos ácido-alcohol resistentes (BAAR) se dio en el 21.1\% de las muestras de heces teñidas con Ziehl Neelsen. Tres pooles resultaron positivos con IS900 y tres con 16S. La secuenciación indicó la presencia de tres especies de bacterias: MAP, Mycobacterium abscessus y Dietzia timorensis. Se identifica por primera vez la presencia de MAP en el departamento de Sucre.
\end{abstract}

Palabras clave: Mycobacterium avium subsp. paratuberculosis; PCR; enfermedades del ganado; Sincelejo; paratuberculosis

\section{Abstract}

The objective of the study was to determine the presence of Mycobacterium avium subsp. paratuberculosis (MAP) in cattle faeces from the municipality of Sincelejo, Sucre, Colombia. A direct bacteriological study was performed by microscopy techniques and polymerase chain reaction (PCR) for pools of faeces with two molecular markers, one specific for MAP (IS900) and another based on 16S of the genus Mycobacterium. The presence of acid-fast bacilli occurred in $21.1 \%$ of the faecal samples stained with Ziehl Neelsen. Three pools were positive with IS900 and three with 16S. The sequencing

\footnotetext{
${ }^{1}$ Grupo de Investigaciones Biomédicas, Universidad de Sucre, Sucre, Colombia

${ }^{2}$ Grupo de Investigaciones GENES, Universidad de Córdoba, Córdoba, Colombia

${ }^{3}$ E-mail: libardo.caraballo@unisucre.edu.co
}

Recibido: 5 de enero de 2018

Aceptado para publicación: 26 de junio de 2018 
indicated the presence of three species of bacteria: MAP, Mycobacterium abscessus and Dietzia timorensis. The presence of MAP in the department of Sucre is identified for the first time.

Key words: Mycobacterium avium subsp paratuberculosis; PCR; cattle diseases; Sincelejo; paratuberculosis

\section{INTRODUCCIÓN}

El agente causal de la paratuberculosis o enfermedad de Johne's es un Mycobacterium del complejo avium (MAC), conocido como Mycobacterium avium subsp. paratuberculosis (MAP). Es una bacteria pequeña, ácido alcohol resistente y no tuberculosa (McNees et al., 2015). En el bovino causa una enteritis crónica granulomatosa que se caracteriza por largos periodos de incubación, pérdida de peso, diarrea, caquexia progresiva y muerte (Gilardoni et al., 2016). A nivel económico, MAP induce grandes pérdidas en el sector ganadero, inducidos principalmente por la disminución de la producción de leche, los efectos adversos en el rendimiento reproductivo y el aumento de las tasas de sacrificio (Britton et al., 2016).

Existe un amplio rango de técnicas usadas para detectar la infección por MAP en bovinos, pero su funcionalidad puede variar dependiendo de la etapa de la infección de la enfermedad. Whitlock et al. (2000) describen tres estados de infección con MAP: (1) los bovinos no excretan MAP en las heces y no presentan signos clínicos, (2) los bovinos excretan MAP en las heces, pero no exhiben signos clínicos de la enfermedad, y (3) los bovinos excretan MAP y presentan signos clínicos de la enfermedad (Britton et al., 2016). Es por esto que se debe tener presente la clínica presentada por el bovino para utilizar una prueba diagnóstica que arroje resultados acordes con la infección del animal.

La reacción en cadena de la polimerasa (PCR, por su sigla en inglés) ofrece un método rápido, rentable y de alto rendimiento para evaluar el estado del MAP en una serie de muestras, incluyendo cultivos, heces, tejidos y leche. Esta técnica puede adaptarse para detectar diferentes dianas genéticas, tales como la secuencia de inserción (IS) IS900 o IS1311. La IS900-PCR se utiliza comúnmente para analizar muestras, debido a que es una secuencia con aproximadamente 15 copias dentro del genoma de MAP, lo cual la hace altamente sensible, aunque también puede ser encontrada en otras especies de micobacterias, de allí que sea necesario emplear otro gen diana o blanco al momento de hacer el diagnóstico por PCR.

Conocido el impacto de la ganadería en la economía nacional y los problemas que genera MAP al sector ganadero, se justifica tener métodos de diagnóstico que permitan una detección temprana de hatos con vacas infectadas a fin de desarrollar estrategias que permitan controlar los brotes y pérdidas para los ganaderos. Además, conociendo la relación aparente entre MAP y la enfermedad de Crohn, y que la leche y sus derivados son una vía de transmisión del microorganismo, se requiere desarrollar estrategias de vigilancia para un monitoreo de la leche que sale de los predios ganaderos.

\section{Materiales y Métodos}

\section{Zona de Estudio}

El estudio se realizó en el departamento de Sucre, municipio de Sincelejo, en fincas de los corregimientos de San Antonio, Sabanas de Potrero, Las Huertas, Buena Vista, Buenavística, San Jacinto, vía a Chochó, vía a Las Palmas y La Garita. El municipio de 
Sincelejo tiene un área de $292 \mathrm{~km}^{2}$. Las actividades económicas de mayor importancia son la ganadería, especialmente de vacunos, la agricultura y el comercio. Sincelejo es uno de los municipios ganaderos más importantes en todo el departamento (IGAC, 1996).

\section{Tamaño de Muestra}

El tamaño de muestra se calculó con base a una población de 31376 de vacunos en el municipio de Sincelejo, con un error del $5 \%$, un nivel de confianza del $95 \%$ y considerando que $50 \%$ de la población se encuentra en riesgo de padecer la enfermedad. El número de muestra resultante fue de 268 individuos. Las fincas se agruparon por conglomerados y de cada grupo se hizo un muestreo aleatorio simple.

\section{Recolección de Muestras}

Se muestrearon bovinos de todas las edades, con previa firma de un consentimiento informado por los dueños o encargados de los predios, donde se explicaban los fines, beneficios, objetivos del estudio y potenciales riesgos según lo establecido en la resolución 08430 de 1993 del Ministerio de Salud de Colombia.

Se recolectaron muestras de heces directamente del recto de cada animal, introduciendo el brazo protegido con un guante desechable que lo cubría hasta por encima del codo. A unos $20 \mathrm{~cm}$ de haber introducido el brazo se cogía la muestra y al sacar el brazo se invertía el guante para evitar contacto de las heces con el medio exterior. Se sellaba y mantenía a $4{ }^{\circ} \mathrm{C}$ en una caja de poliestireno expandido con gel refrigerante hasta su procesamiento.

\section{Identificación de Bacilos Ácido Alcohol Resistentes (BAAR)}

Se realizaron frotis de heces, se colorearon con el reactivo de Ziehl Neelsen y fueron observados al microscopio a $100 \mathrm{X}$ para la identificación de bacilos ácido-alcohol resistentes (Bernardelli, 2000).

\section{Extracción de ADN y PCR}

Las muestras de heces fueron agrupadas en pooles de cinco individuos según la finca donde fueron colectadas. A cada pool se le hizo extracción de ADN empleando el método desarrollado por Garrido et al. (2000), con algunas modificaciones. En breve, del método indicado solo se utilizó la parte correspondiente a la precipitación del ADN con SDS al 5\% y lavados consecutivos con PBS 1X. Seguido a esto, se procedió a extraer el ADN con TRIzol® Reagent, siguiendo las indicaciones del inserto del reactivo. Los extractos de ADN fueron cuantificados en un NanoDrop 2000 y diluidos $1 / 10$ para la reacción de PCR (Cuadro 1). Los productos fueron corridos en un gel de agarosa al 1.8\%, teñidos con Gel Start durante 40 minutos a $80 \mathrm{v}$. Luego fueron visualizados haciendo uso de un fotodocumentador. Una muestra se consideró positiva al presentar el tamaño del fragmento génico de interés $\mathrm{y}$, además, por ser similar al control positivo utilizado (ADN de MAP aislado de un bovino en Antioquía). Como control negativo se utilizó agua ultra pura. Los productos PCR positivos fueron enviados a secuenciar a Macrogen (Corea del Sur).

Los resultados de la secuenciación fueron analizados con el programa MEGA 6 (Tamura et al., 2013) y la comparación de homología de secuencias se realizó con la base de datos GenBank.

Al momento de la toma de muestras se tomaron datos sobre el sexo, edad, ocurrencia de cuadros diarreicos y estado físico del animal, tipo de explotación ganadera, manejo de las excretas, fuentes de agua para el consumo animal, así como la población total y destino de la producción, con la finalidad de evaluar la asociación de estos factores con la presencia de la enfermedad (SánchezVillalobos et al., 2009). 
Cuadro 1. Cebadores y programas utilizados para las pruebas de PCR

\begin{tabular}{|c|c|c|c|c|}
\hline Cebadores & Secuencias & $\begin{array}{l}\text { Tamaño del } \\
\text { producto }\end{array}$ & $\begin{array}{l}\text { Concentraciones y } \\
\text { volúmenes finales }\end{array}$ & Programas \\
\hline $\begin{array}{l}\text { IS900 } \\
\text { (Sanderson et } \\
\text { al., 1992; } \\
\text { Stabel et al., } \\
\text { 2004) }\end{array}$ & $\begin{array}{l}\text { IS900F 5'-CCG CTA ATT GAG } \\
\text { t AGA TGC GAT TGG-3' } \\
\text { IS900R 5'-AAT CAA CTC CAG } \\
\text { CAG CGC GGC CTC G-3' }\end{array}$ & $229 \mathrm{pb}$ & $\begin{array}{l}\text { Mix } 25 \mu \mathrm{l}, \\
\text { buffer } 10 \mathrm{X} 2.5 \mu \mathrm{l}, \\
\mathrm{MgCl}_{2} \text { a } 1 \mathrm{mM} 1 \mu \mathrm{l}, \\
\text { dNTPs a } 0.2 \mathrm{mM} 2.5 \mu \mathrm{l} \text {, } \\
\text { cebador a } 10 \mathrm{pmol}(\mathrm{c} / \mathrm{u}) \\
0.25 \mu \mathrm{l}, \\
\text { Taq } 1 \mathrm{U} / \mu \mathrm{l} 0.2 \mu \mathrm{l}, \\
\text { ADN } 3 \mu \mathrm{l}\end{array}$ & $\begin{array}{l}5^{\prime} \text { a } 94{ }^{\circ} \mathrm{C}, \\
35 \text { ciclos } \\
\left(45^{\prime \prime} \text { a } 94{ }^{\circ} \mathrm{C},\right. \\
30 \text { a } 60^{\circ} \mathrm{C}, \\
\left.45^{\prime \prime} \text { a } 72{ }^{\circ} \mathrm{C}\right) \\
\text { y } 10^{\prime} \text { a } 72^{\circ} \mathrm{C}\end{array}$ \\
\hline $\begin{array}{l}\text { 16S rRNA } \\
\text { (Tevere } \text { et } \\
\text { al., 1996; } \\
\text { Hsiao et al., } \\
\text { 2003) }\end{array}$ & $\begin{array}{l}\text { KY } 75 \text { 5'GCC CGT ATC GCC } \\
\text { CGC ACG CTC ACA-3' } \\
\text { KY } 18 \text { 5'CAC ATG CAA GTC } \\
\text { GAA CGG AAA GG-3' }\end{array}$ & $565-590 \mathrm{pb}$ & $\begin{array}{l}\text { Mix } 25 \mu \mathrm{l}, \\
\text { buffer } 10 \mathrm{X} 2.5 \mu \mathrm{l}, \\
\mathrm{MgCl}_{2} \text { a } 1.5 \mathrm{mM} 1.5 \mu \mathrm{l}, \\
\text { dNTPs a } 0.2 \mathrm{mM} 2.5 \mu \mathrm{l} \text {, } \\
\text { cebador a } 10 \mathrm{pmol}(\mathrm{c} / \mathrm{u}) \\
0.25 \mu \mathrm{l}, \\
\text { Taq } 1 \mathrm{U} / \mu \mathrm{l} 0.2 \mu \mathrm{l}, \\
\text { ADN } 3 \mu \mathrm{l}\end{array}$ & $\begin{array}{l}5, \text { a } 95^{\circ} \mathrm{C}, \\
35 \text { ciclos } \\
\left(20^{\prime \prime} \text { a } 94^{\circ} \mathrm{C},\right. \\
30 \text { a } 65^{\circ} \mathrm{C}, \\
\left.45^{\prime \prime} \text { a } 72^{\circ} \mathrm{C}\right) \\
\text { y } 10^{\prime} \text { a } 72^{\circ} \mathrm{C}\end{array}$ \\
\hline
\end{tabular}

Cuadro 2. Distribución porcentual de las 26 fincas seleccionadas para el muestreo de Mycobacterium avium subespecie paratuberculosis, según el corregimiento, Municipio de Sincelejo, Colombia

\begin{tabular}{lc}
\hline Corregimiento & Porcentaje \\
\hline Buenavista & 19.2 \\
Buenavística & 7.7 \\
La Garita & 3.8 \\
Las Huertas & 15.4 \\
Sabana del Potrero & 23.1 \\
San Jacinto & 7.7 \\
Vía a Chochó & 19.2 \\
Vía Las Palmas & 3.8 \\
\hline Total & 100 \\
\hline
\end{tabular}

\section{Análisis Estadístico}

Se hizo un análisis descriptivo de los datos, seguido de una prueba exacta de Fisher para determinar posibles asociaciones entre las variables con los resultados del PCR. El valor de significancia utilizado fue de $\mathrm{p}<0.05$.
El análisis fue corrido en el programa InfoStat, versión estudiantil.

\section{Resultados}

Se visitaron 26 fincas correspondientes a ocho corregimientos del municipio de Sincelejo (Cuadro 2), tomándose muestras de heces a 275 bovinos. Las muestras se agruparon en 57 pooles. La mayor proporción de animales estuvo en los rangos de $>1$ y $3(36 \%)$ y $>3$ y 5 (40\%) (Figura 1). Así mismo, el $98.9 \%(272 / 275)$ fueron hembras, dado que se trabajó con ganado lechero.

Del total de las muestras coloreadas con la técnica de Zielh Neelsen, 58 de 275 (21.1\%) resultaron sospechosas al visualizarse la presencia de los bacilos de una tonalidad rosa en las placas (Figura 2).

La mayor cantidad de individuos con sospecha de excreción de MAP en las heces provenían de fincas de los corregimientos de Buenavista (16/61), Sabanas del Potrero (13/ 


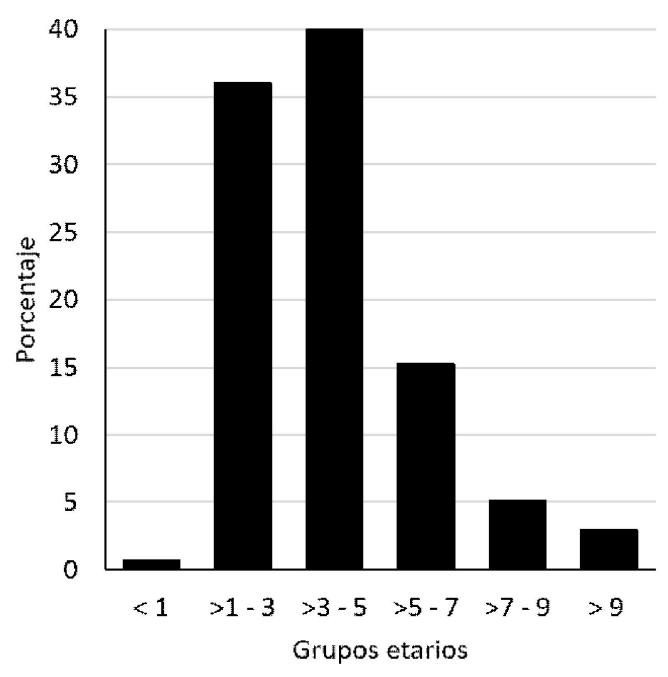

Figura 1. Distribución porcentual de los grupos etarios de bovinos muestreados en el Municipio de Sincelejo

52) y Las Huertas (10/33). Así mismo, la mayor cantidad de individuos sospechosos por Ziehl Neelsen se encontraba en el rango de edad $>1$ y 3 ( $8.4 \%)$.

Mediante el analisis por PCR utilizando los cebadores del la secuencia de inserción IS900 se detectó ADN de MAP en tres pooles de heces que correspondian a tres fincas $(11.5 \%, 3 / 26)$, dos del corregimiento de Buenavista y una de la Vía a Chochó (Figura 3). Por otro lado, al utilizar los cebadores basados en el gen 16S de Mycobacterium se detectó ADN en tres muestras $(11.5 \%, 3 / 26)$ distintas a las obtenidas con IS900 (Figura 4).

Las muestras detectadas con IS900 indican que, en efecto, el ADN extraido correspondía a MAP, indicando la presencia de la bacteria en el municipio de Sincelejo y por extensión en el departamento de Sucre; hecho que fue sustentado por los resultados de la secuenciación, donde las secuencias obtenidas fueron homólogas a secuencias registradas en la base de datos GenBank con un $99 \%$ de identidad. Por otro lado, la secuenciación del segmento del gen $16 \mathrm{~S}$ indicó la presencia de Mycobacterium abscessus y Dietzia timorensis, dos especies de bacterias dentro del orden Actinomycetales.

El análisis de asociación de las variables registradas durante la toma de las muestras con la detección de MAP en heces no mostró relación estadística.

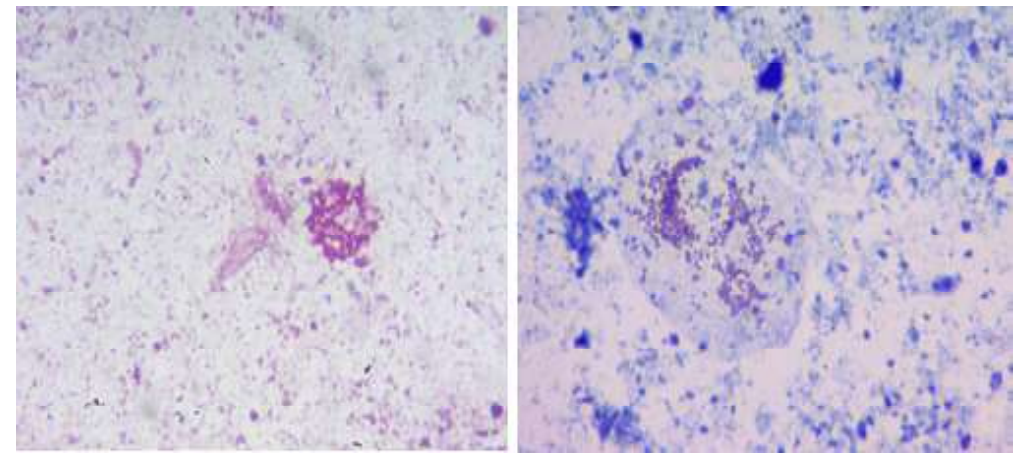

Figura 2. Bacilos ácido alcohol-resistentes en frotis de heces de bovinos del municipio de Sincelejo. $100 \mathrm{X}$ 


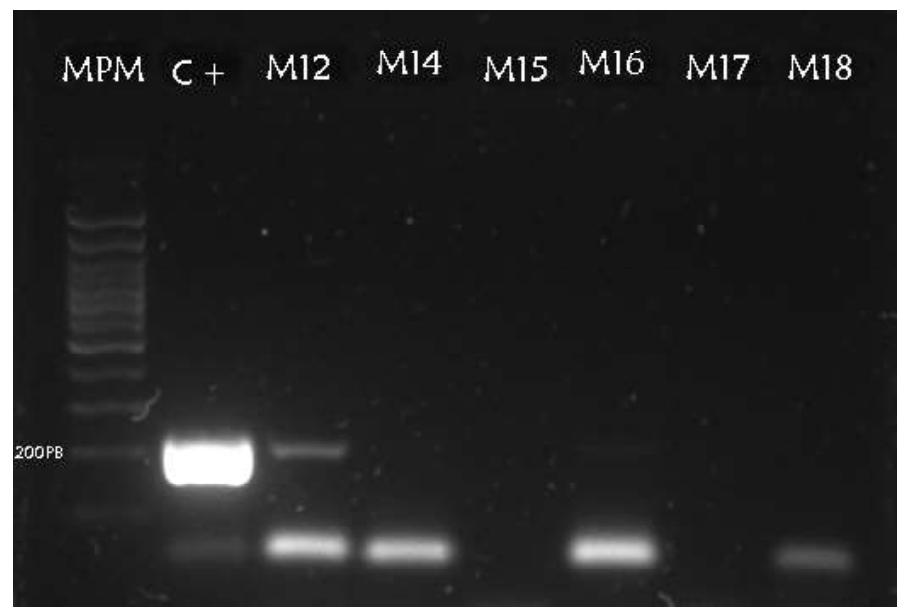

Figura 3. Electroforesis en gel de Agarosa para detección de MAP. MPM: marcador de peso molecular de 100pb; $\mathrm{C}+$ : control positivo; $\mathrm{M}$ : muestras

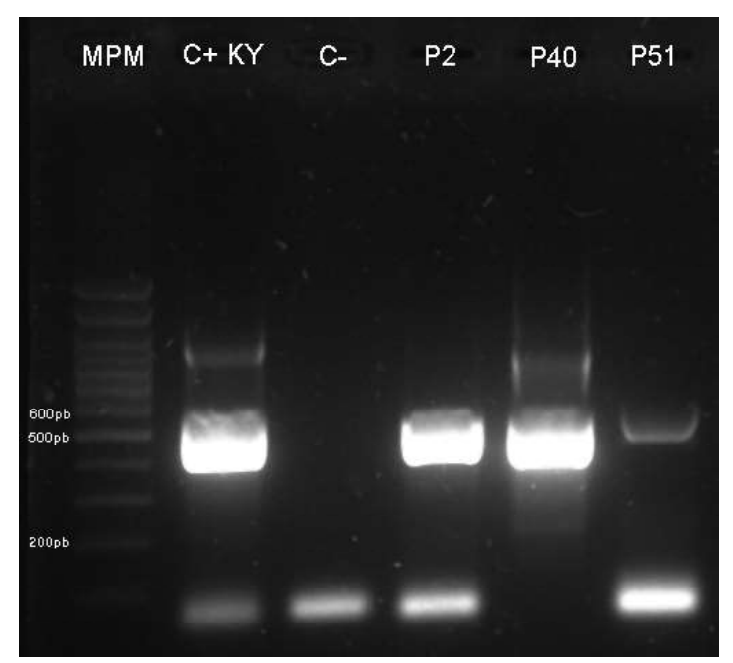

Figura 4. Electroforesis en gel de Agarosa para detección del gen 16S de Mycobacterium. MPM: marcador de peso molecular de $100 \mathrm{pb}$; $\mathrm{C}+$ : control positivo; C-: control negativo; P: muestras

\section{Discusión}

La paratuberculosis se conoce en Colombia desde 1924, cuando fue diagnosticada por primera vez en la hacienda El Hato en Usme, cerca de Bogotá. Posteriormente, el Instituto Colombiano Agropecuario (ICA) reportó casos entre 1986 y 1993 en el departamento del Meta (Góngora y Villamil, 1999).
En años más recientes, se han reportado casos en el Municipio de San Pedro de los Milagros, Antioquia, en una de las fincas lecheras del departamento (Zapata Restrepo et al., 2008), mientras que en Nariño se encontró un seroprevalencia del 94\% (Benavides et al., 2015); sin embargo, aún no existe un pragrama de trabajo sólido que ayude a comprender el comportamiento de la paratuberculosis en los hatos ganaderos del país. 
En este estudio se determinó por primera vez la presencia de MAP en heces de bovinos del departamento de Sucre, con un acercamiento inicial mediante la tinción de Ziehl Neelsen, donde el $21.1 \%$ de las muestras sugerian la presencia de BAAR. Dichos resultados son consistentes con los trabajos de Méndez et al. (2013), quienes obtuvieron $8.2 \%(6 / 136)$ de muestras de heces con la presencia de bacilos sugestivos de MAP, y con el estudio de Weber et al. (2009) con $27.3 \%(538 / 1968)$ de muestras positivas por Ziehl Neelsen.

Hasta este punto, se tenía indicios de la presencia de MAP en fincas ganaderas del municipio de Sincelejo, lo cual enciende las alarmas debido al problema de salud pública que es la paratuberculosis. Los resultados llevan a sospechar que a través de la leche también se esté excretando la bacteria, al encontrarse que la mayoría de vacas sospechosas se encuentran entre $>1$ y 3 años de edad, que es la edad promedio en que las vacas inician su mayor producción lechera (Bolívar et al., 2009). Sin embargo, solo se puede hablar de bovinos con sospecha de estar excretando MAP, dado que la técnica de tinción de Ziehl Neelsen solo produce un diagnóstico presuntivo debido a la existencia de otros grupos de bacterias con características ácido alcohol-resistentes como Nocardia, Clostridium y Actinobacterias (Zimmer et al., 1999; Negroni, 2009; Weber et al., 2009; Méndez Olvera et al., 2013).

Con IS900-PCR se pudo identificar los predios con presencia real de MAP en vacas en estado subclínico. El 11.5\% de frecuencia de infección fue relativamente similar al $29.5 \%$ (7/27) encontrado por Correa (2016) utilizando un juego de cebadores de la IS900. Así mismo, Douarre et al. (2010) detectaron ADN de MAP en el 36.2\% (105/290) de muestras colectadas en siete fincas de Irlanda con antecedentes clínicos de enfermedad de Johne.
Se reconoce que el uso directo de la PCR en ADN extraído de material fecal facilita el diagnóstico de la enfermedad de Johne en comparación con el cultivo ( 3 días vs. 1422 semanas); además, es es una prueba relativamente económica barata que permite evaluar un número representativo de animales de un hato (Douarre et al., 2010). Por ello, es de suponer que al detectar ADN de MAP en un individuo, se debe considerar en estado infeccioso y, por ende, todo el predio se debe considerar en riesgo.

La mayoria de vacas en las fincas positivas a MAP estaban entre un rango de edad de $>3$ y $<7$ años, lo que concuerda con la historia natural de la enfermedad, considerando que el periodo de incubación de la infección es de aproximadamente 5 años (Salgado et al., 2013; Benavides et al., 2015). Sin embargo, no se halló asociación sigificativa con esta variable, dado posiblemente porque en este periodo de la enfermedad hay poca evidencia de la excresión de MAP (Windsor y Whittington, 2010).

\section{Conclusiones}

Mycobacterium avium subespecie paratuberculosis (MAP) está presente en el municipio de Sincelejo y por extensión en el departamento de Sucre.

\section{Agradecimientos}

Los autores agradecen a los propietarios de las fincas, al veterinario de FEDEGAN Fredy Beleñó, a Nathalia María del Pilar Correa y al Dr. Jorge Fernández Silva del grupo CENTAURO de la Universidad de Antioquia, al proyecto Formación de Capacidades en Ciencia, Tecnología e Innovación en el departamento de Sucre, Caribe, a la gobernación de Sucre, al GRUBIODEQ de la Universidad de Cordóba y al Grupo Biomédicas de la Universidad de Sucre. 


\section{Literatura Citada}

1. Benavides B, Arteaga AV, Montezuma CA. 2015. Estudio epidemiológico de paratuberculosis bovina en hatos lecheros del sur de Nariño, Colombia. Rev Med Vet 31: 57-66.

2. Bernardelli A. 2000. Manual de procedimiento técnico: deagnóstico de paratuberculosis. México: SENASA. 74 p.

3. Bolivar DM, Echeverry JJ, Restrepo LF, Cerón MF. 2009. Productividad de vacas Jersey, Holstein y Jersey x Holstein en una zona de bosque húmedo montano bajo (Bh-MB). Livestock Res Rural Dev 21(6). [Internet]. Disponible en: http://www.lrrd.org/lrrd21/6/boli21080.htm

4. Britton LE, Cassidy JP, O'Donovan J, Gordon SV, Markey B. 2016. Potential application of emerging diagnostic techniques to the diagnosis of bovine Johne's disease (paratuberculosis). Vet J 209: 3239. doi: 10.1016/j.tvj1.2015.-10.033

5. Correa N. 2016. Diagnosis and risk factors of Mycobacterium avium subsp. paratuberculosis (MAP) in dairy herds of the Northern Region of Antioquia, Colombia. Tesis de Maestría. Colombia: Univ. de Antioquia. $164 \mathrm{p}$.

6. Douarre P, Cashman W, Buckley J, Coffey A, O'Mahony J. 2010. Isolation and detection of Mycobacterium avium subsp. paratuberculosis (MAP) from cattle in Ireland using both traditional culture and molecular based methods. Gut Pathog 2: 11-18. doi: 10.1186/17574749-2-11

7. Garrido JM, Cortabarria N, Oguiza JA, Aduriz G, Juste RA. 2000. Use of a PCR method on fecal samples for diagnosis of sheep paratuberculosis. Vet Microbiol 77: 379-386. doi: 10.1016/ S0378-1135(00)00323-0

8. Gilardoni LR, Fernández B, Morsella C, Mendez L, Jar AM, Paolicchi FA, Mundo SL. 2016. Mycobacterium paratuberculosis detection in cow's milk in Argentina by immunomagnetic separation-PCR. Braz J Microbiol 47: 506-512. doi: 10.1016/j.bjm.2016.01.013

9. Góngora A, Villamil L. 1999. La paratuberculosis bovina desde la óptica de la salud pública. Holstein Colomb 147: 44-48.

10. Hsiao PF, Tzen CY, Chen HC, Su HY. 2003. Polymerase chain reaction based detection of Mycobacterium tuberculosis in tissues showing granulomatous inflammation without demonstrable acidfast bacilli. Int J Dermat 42: 281-286. doi: 10.1046/j.1365-4362.2003.01461.x

11. [IGAC] Diccionario geográfico de Colombia. 1996. Bogotá: Instituto geográfico Agustin Codazzi. $620 \mathrm{p}$.

12. McNees AL, Markesich D, Zayyani NR, Graham DY. 2015. Mycobacterium paratuberculosis as a cause of Crohn's disease. Expert Rev Gastroent 9: 1523-1534. doi: 10.1586/17474124.2015.1093931

13. Méndez ET, Ramírez IN, Rojas N, Olivares JL, Martínez D. 2013. Detección de Mycobacterium avium paratuberculosis en caprinos ubicados en una zona semi-árida en el municipio de Tecozautla Hidalgo. Rev Salud Anim 35: 182-188.

14. Negroni M. 2009. Microbiología estomatológica: fundamentos y guía práctica. $2^{\circ}$ ed. Médica Panamericana. $656 \mathrm{p}$.

15. Salgado M, Steuer P, Troncoso E, Collins MT. 2013. Evaluation of PMSPCR technology for detection of Mycobacterium avium subsp. paratuberculosis directly from bovine fecal specimens. Vet Microbiol 167: 725-728. doi: 10.1016/j.vetmic.2013.09.009

16. Sánchez-Villalobos A, Arráiz-Rodríguez N, Becerra-Ramírez L, Faria N, Montero-Urdaneta M, Oviedo-Bustos A, Pino-Ramírez D. 2009. Infección por Mycobacterium avium subsp. paratuberculosis en un rebaño criollo limonero. Rev Cient LUZ 19: 555: 565. 
17. Sanderson JD, Moss MT, Tizard ML, Hermon-Taylor J. 1992. Mycobacterium paratuberculosis DNA in Crohn's disease tissue. Gut 33: 890-896. doi: 10.1136/gut.33.7.890

18. Stabel J, Bosworth T, Kirkbride T, Forde R, Whitlock R. 2004. A simple, rapid, and effective method for the extraction of Mycobacterium paratuberculosis DNA from fecal samples for polymerase chain reaction. J Vet Diagn Invest 16: 22-30. doi: 10.1177/104063870401600105

19. Tamura K, Stecher G, Peterson D, Filipski A, Kumar S. 2013. MEGA6: Molecular evolutionary genetics analysis version 6.0. Mol Biol Evol 30: 2725-2729. doi: $10.1093 / \mathrm{molbev} / \mathrm{mst} 197$

20. Tevere VJ, Hewitt PL, Dare A, Hocknell P, Keen A, Spadoro JP, Young KK. 1996. Detection of Mycobacterium tuberculosis by PCR amplification with pan-Mycobacterium primers and hybridization to an $M$. $t u$ berculosis-specific probe. J Clin Microbiol 34: 918-923.

21. Weber MF, Verhoeff J, van Schaik G, van Maanen C. 2009. Evaluation of Ziehl-Neelsen stained faecal smear and
ELISA as tools for surveillance of clinical paratuberculosis in cattle in the Netherlands. Prev Vet Med 92: 256-266. doi: 10.1016/j.prevetmed.2009.08.017

22. Whitlock RH, Wells SJ, Sweeney RW, van Tiem J. 2000. ELISA and fecal culture for paratuberculosis (Johne's disease): sensitivity and specificity of each method. Vet Microbiol 77: 387-398. doi: 10.1016/S0378-1135(00)00324-2

23. Windsor PA, Whittington RJ. 2010. Evidence for age susceptibility of cattle to Johne's disease. Vet J 184: 37-44. doi: 10.1016/j.tvj1.2009.01.007

24. Zapata MM, Rodas JD, Maldonado JG 2008. Paratuberculosis bovina: ¿conocemos la situación real de la enfermedad en la ganadería colombiana? Rev Colomb Cienc Pec 21: 429-435.

25. Zimmer K, Drager KG, Klawonn W, Hess RG. 1999. Contribution to the diagnosis of Johne's disease in cattle. Comparative studies on the validity of Ziehl-Neelsen staining, faecal culture and a commercially available DNA-Probe test in detecting Mycobacterium paratuberculosis in faeces from cattle. $\mathrm{Zbl}$ Vet Med B 46: 137-140. doi: 10.1111/ j.0931-1793.1999.00214.x 